\title{
OSCILLATION THEOREMS FOR SECOND ORDER DIFFERENCE EQUATIONS WITH NEGATIVE NEUTRAL TERM
}

\author{
DEVARAJULU SEGHAR, ETHIRAJU THANDAPANI AND SANDRA PINELAS
}

\begin{abstract}
In this paper we obtain some new oscillation criteria for the neutral difference equation

$$
\Delta\left(a_{n}\left(\Delta\left(x_{n}-p_{n} x_{n-k}\right)\right)\right)+q_{n} f\left(x_{n-l}\right)=0
$$

where $0 \leq p_{n} \leq p<1, q_{n}>0$ and $l$ and $k$ are positive integers. Examples are presented to illustrate the main results. The results obtained in this paper improve and complement to the existing results.
\end{abstract}

\section{Introduction}

Consider the second order neutral difference equation of the form

$$
\Delta\left(a_{n}\left(\Delta\left(x_{n}-p_{n} x_{n-k}\right)\right)\right)+q_{n} f\left(x_{n-l}\right)=0, n \in \mathbb{N}\left(n_{0}\right)
$$

where $\mathbb{N}\left(n_{0}\right)=\left\{n_{0}, n_{0}+1, \ldots\right\}, \quad n_{0}$ is a nonnegative integer, subject to the following conditions:

$\left(H_{1}\right)\left\{a_{n}\right\}$ is a positive real sequence with $\sum_{n=n_{0}}^{\infty} \frac{1}{a_{n}}<\infty$;

$\left(H_{2}\right)\left\{p_{n}\right\}$ is a real sequence with $0 \leq p_{n} \leq p<1$ for all $n \in \mathbb{N}\left(n_{0}\right)$;

$\left(H_{3}\right)\left\{q_{n}\right\}$ is a positive real sequence for all $n \in \mathbb{N}\left(n_{0}\right)$;

$\left(H_{4}\right) l$ and $k$ are positive integers;

( $\left.H_{5}\right) \quad f: \mathbb{R} \rightarrow \mathbb{R}$ is a continuous function with $u f(u)>0$ for $u \neq 0$, and there exists a constant $M>0$ such that $\frac{f(u)}{u^{\alpha}}>M$ for all $u \neq 0$, where $\alpha$ is a ratio of odd positive integers.

Received January 10, 2015, accepted June 6, 2015.

2010 Mathematics Subject Classification. 39A11.

Key words and phrases. Oscillation, second-order, neutral difference equation, negative neutral term. Corresponding author: E. Thandapani. 
Let $\theta=\max \{k, l\}$. By a solution of equation (1.1), we mean a real sequence $\left\{x_{n}\right\}$ defined for $n \geq n_{0}-\theta$ and satisfying equation (1.1) for all $n \in \mathbb{N}\left(n_{0}\right)$. A solution of equation (1.1) is said to be oscillatory if it is neither eventually positive nor eventually negative, and it is nonoscillatory otherwise.

From a review of literature, it is known that there are many results available on the oscillatory and asymptotic behavior of solutions of equation (1.1) when the neutral term is nonnegative, i.e., $p_{n} \leq 0$; see for example $[1,2,3,9,15]$ and the references cited therein. However, there are few results available on the oscillatory behavior of solutions of equation (1.1) when the neutral term is negative; see, for example $[4,6,7,8,11,12,13,14,16,17]$ and the references therein.

In [1], we see that the oscillatory behavior of the equation

$$
\Delta^{2}\left(x_{n}-p x_{n-k}\right)+q_{n} x_{n-l}=0, n \in \mathbb{N}\left(n_{0}\right),
$$

is discussed and in [14], the authors studied the oscillatory and asymptotic behavior of equation

$$
\Delta\left(a_{n}\left(\Delta\left(x_{n}-p_{n} x_{n-k}\right)\right)\right)+q_{n} x_{n-l}^{\alpha}=0, n \in \mathbb{N}\left(n_{0}\right),
$$

with $\sum_{n=n_{0}}^{\infty} \frac{1}{a_{n}}=\infty$. The results obtained for equations (1.2) and (1.3) has been improved and generalized by other authors. We mention Thandapani et al.[12] studied the oscillation of

$$
\Delta\left(a_{n}\left(\Delta\left(x_{n}-p_{n} x_{n-k}\right)\right)^{\alpha}\right)+q_{n} f\left(x_{n-l}\right)=0,
$$

under the conditions

$$
\frac{f(u)}{u^{\alpha}} \geq M, \text { and } \sum_{n=n_{0}}^{\infty} \frac{1}{a_{n}^{\frac{1}{\alpha}}}=\infty .
$$

In all the results, the authors assumed that either $a_{n}=1$ or $\sum_{n=n_{0}}^{\infty} \frac{1}{a_{n}}=\infty$, and as far as the authors knowledge there are no worthwhile results available in the literature when $\sum_{n=n_{0}}^{\infty} \frac{1}{a_{n}}<$ $\infty$ for the equation (1.1). This observation motivated us to study the oscillatory behavior of equation (1.1) when condition $\left(H_{1}\right)$ is satisfied. In Section 2, we obtain some new sufficient conditions for the oscillation of all solutions of equation (1.1), and in Section 3, we provide some examples to illustrate the main results. Thus the results presented in this paper improve and complement to those established in $[4,11,12,13,14,16,17]$.

\section{Oscillation results}

Throughout this paper, we use the following notation without further mention:

$$
z_{n}=x_{n}-p_{n} x_{n-k}
$$




$$
A_{n}=\sum_{s=n_{0}}^{n-1} \frac{1}{a_{s}}, \quad \text { and } \quad B_{n}=\sum_{s=n}^{\infty} \frac{1}{a_{s}} .
$$

Remark 2.1. Without loss of generality, we can deal only with positive solutions of equation (1.1), since the proof for the other case is similar.

We begin with the following lemma.

Lemma 2.1. Let $\left\{x_{n}\right\}$ be an eventually positive solution of equation (1.1). Then one of the following three cases holds for all sufficiently large $n$ :

(I) $z_{n}>0, a_{n} \Delta z_{n}>0, \Delta\left(a_{n} \Delta z_{n}\right) \leq 0$;

(II) $z_{n}>0, a_{n} \Delta z_{n}<0, \Delta\left(a_{n} \Delta z_{n}\right) \leq 0$;

(III) $z_{n}<0, a_{n} \Delta z_{n}>0, \Delta\left(a_{n} \Delta z_{n}\right) \leq 0$.

Proof. Assume that $x_{n-\theta}>0$ for $n \geq N \in \mathbb{N}\left(n_{0}\right)$. Then by the condition $\left(H_{3}\right)$, we have from equation (1.1) that $\Delta\left(a_{n} \Delta z_{n}\right) \leq 0$ for all $n \geq N$. Hence $\left\{z_{n}\right\}$ and $\left\{a_{n} \Delta z_{n}\right\}$ are eventually of one sign for all $n \geq N$. Thus $\left\{z_{n}\right\}$ satisfying one of the following four cases for all $n \geq N$ :

(I) $z_{n}>0, a_{n} \Delta z_{n}>0, \Delta\left(a_{n} \Delta z_{n}\right) \leq 0$;

(II) $z_{n}>0, a_{n} \Delta z_{n}<0, \Delta\left(a_{n} \Delta z_{n}\right) \leq 0$;

(III) $z_{n}<0, a_{n} \Delta z_{n}>0, \Delta\left(a_{n} \Delta z_{n}\right) \leq 0$;

(IV) $z_{n}<0, a_{n} \Delta z_{n}<0, \Delta\left(a_{n} \Delta z_{n}\right) \leq 0$.

Now, we shall show that case (IV) cannot happen. If so, then we have $\lim _{n \rightarrow \infty} z_{n}=-\infty$. From the definition of $z_{n}$, we obtain $x_{n}>\left(\frac{-z_{n+k}}{p}\right)$, and therefore $\limsup _{n \rightarrow \infty} x_{n}=\infty$. Thus there exists a subsequence $\left\{n_{j}\right\}$ of positive integers such that $\lim _{j \rightarrow \infty} n_{j}=\infty$ and $x_{n_{j}}=\max _{n_{0} \leq n \leq n_{j}} x_{n} \rightarrow \infty$ as $j \rightarrow \infty$. Then

$$
z_{n_{j}}=x_{n_{j}}-p_{n_{j}} x_{n_{j}-k} \geq x_{n_{j}}-p x_{n_{j}}=x_{n_{j}}(1-p) \rightarrow \infty
$$

as $j \rightarrow \infty$, a contradiction. This completes the proof.

Lemma 2.2. If $\left\{x_{n}\right\}$ is an eventually positive solution of equation (1.1) such that case (I) holds, then

$$
x_{n} \geq z_{n} \geq A_{n} a_{n} \Delta z_{n}, \quad n \geq N \in \mathbb{N}\left(n_{0}\right),
$$

and $\left\{\frac{z_{n}}{A_{n}}\right\}$ is eventually strictly decreasing. 
Proof. The proof is similar to that of Lemma 2 in [12], and hence the details are omitted.

Lemma 2.3. If $\left\{x_{n}\right\}$ is an eventually positive solution of equation (1.1) such that case (II) holds, then

$$
x_{n} \geq z_{n} \geq-B_{n} a_{n} \Delta z_{n}, \quad n \geq N \in \mathbb{N}\left(n_{0}\right) .
$$

Proof. From the definition of $z_{n}$, it is clear that $x_{n} \geq z_{n}$ for all $n \geq N$. Since $a_{n} \Delta z_{n}$ is nonincreasing, we have

$$
a_{s} \Delta z_{s} \leq a_{n} \Delta z_{n}, s \geq n \geq N \text {. }
$$

Dividing the last inequality by $a_{s}$ and then summing it from $n$ to $j$, we obtain

$$
z_{j+1} \leq z_{n}+a_{n} \Delta z_{n} \sum_{s=n}^{j} \frac{1}{a_{s}}, j \geq n \geq N .
$$

Letting $j \rightarrow \infty$, we have

$$
0 \leq z_{n}+B_{n} a_{n} \Delta z_{n}, n \geq N
$$

This completes the proof.

Theorem 2.1. Assume that $\alpha=1$ and $l>k$. If

$$
\begin{aligned}
& \limsup _{n \rightarrow \infty} \sum_{s=n-l+k}^{n-1} \frac{1}{a_{s}} \sum_{t=s}^{n-1} q_{t}>\frac{p}{M}, \\
& \liminf _{n \rightarrow \infty} \sum_{s=n-l}^{n-1} q_{s}\left(A_{s-l}+p_{s-l} A_{s-l-k}\right)>\frac{1}{M}\left(\frac{l}{l+1}\right)^{l+1},
\end{aligned}
$$

and

$$
\sum_{n=n_{0}}^{\infty}\left[M q_{n} B_{n+1}-\frac{1}{4 a_{n} B_{n+1}}\right]=\infty
$$

then every solution of equation (1.1) is oscillatory.

Proof. Assume that there exists a nonoscillatory solution $\left\{x_{n}\right\}$ of equation (1.1), say, $x_{n}>0$ and $x_{n-\theta}>0$ for all $n \geq N \in \mathbb{N}\left(n_{0}\right)$, where $N$ is chosen so that all three cases of Lemma 2.1 hold for all $n \geq N$.

Case I: From (2.1), we have

$$
x_{n} \geq z_{n}+p_{n} z_{n-k} \geq\left(1+p_{n} \frac{A_{n-k}}{A_{n}}\right) z_{n}, n \geq N,
$$

where we have used $\left\{z_{n} / A_{n}\right\}$ is decreasing. Using (2.7) and ( $\left.H_{5}\right)$ in equation (1.1), we obtain

$$
\Delta\left(a_{n} \Delta z_{n}\right)+M q_{n}\left(1+p_{n-l} \frac{A_{n-l-k}}{A_{n-l}}\right) z_{n-l} \leq 0, n \geq N .
$$


From (2.2) and (2.8), we have

$$
\Delta\left(a_{n} \Delta z_{n}\right)+M q_{n}\left(A_{n-l}+p_{n-l} A_{n-l-k}\right) a_{n-l} \Delta z_{n-l} \leq 0, n \geq N .
$$

Let $w_{n}=a_{n} \Delta z_{n}$. Then $w_{n}>0$ and $\left\{w_{n}\right\}$ is an eventually positive solution of the inequality

$$
\Delta w_{n}+M q_{n}\left(A_{n-l}+p_{n-l} A_{n-l-k}\right) w_{n-l} \leq 0 .
$$

But by Theorem 7.6.1 of [5], and (2.5), the inequality (2.10) has no eventually positive solution, a contradiction.

Case II: Define

$$
w_{n}=\frac{a_{n} \Delta z_{n}}{z_{n}}, n \geq N .
$$

Then $w_{n}<0$ for all $n \geq N$. From (2.3) and (2.11), we have

$$
-1 \leq B_{n} w_{n} \leq 0, n \geq N
$$

From the equations (1.1), (2.1) and $\left(H_{5}\right)$, we have

$$
\Delta\left(a_{n} \Delta z_{n}\right)+M q_{n} z_{n-l} \leq 0, n \geq N .
$$

From (2.11) and (2.13), we obtain

$$
\begin{aligned}
\Delta w_{n} & \leq-M q_{n} \frac{z_{n-l}}{z_{n+1}}-\frac{a_{n}\left(\Delta z_{n}\right)^{2}}{z_{n} z_{n+1}} \\
& \leq-M q_{n}-\frac{w_{n}^{2}}{a_{n}}, n \geq N,
\end{aligned}
$$

where we have used $\left\{z_{n}\right\}$ is positive decreasing and $l$ is a positive integer. Multiplying (2.14) by $B_{n+1}$ and then summing it from $N$ to $n-1$, we have

$$
\sum_{s=N}^{n-1} B_{s+1} \Delta w_{s}+\sum_{s=N}^{n-1} M B_{s+1} q_{s}+\sum_{s=N}^{n-1} B_{s+1} \frac{w_{s}^{2}}{a_{s}} \leq 0 .
$$

Using summation by parts formula in the first term of (2.15), and then rearranging we obtain

$$
B_{n} w_{n}-B_{N} w_{N}+\sum_{s=N}^{n-1} M B_{s+1} q_{s}+\sum_{s=N}^{n-1}\left(\frac{w_{s}}{a_{s}}+\frac{w_{s}^{2}}{a_{s}} B_{s+1}\right) \leq 0 .
$$

Using completing the square in the fourth term of the last inequality and then using (2.12), we obtain

$$
\sum_{s=N}^{n-1}\left(M B_{s+1} q_{s}-\frac{1}{4 a_{s} B_{s+1}}\right) \leq B_{N} w_{N}-B_{n} w_{n} \leq B_{N} w_{N}+1 .
$$


Letting $n \rightarrow \infty$ in the last inequality, we obtain a contradiction with (2.6).

Case III: From (2.1) and $\left(\mathrm{H}_{2}\right)$, we have

$$
x_{n-k}>\left(\frac{-z_{n}}{p}\right)
$$

Using $\left(H_{5}\right)$ and (2.16) in equation (1.1), we obtain

$$
\Delta\left(a_{n} \Delta z_{n}\right)-\frac{M}{p} q_{n} z_{n-l+k} \leq 0, n \geq N .
$$

Summing (2.17) from $s$ to $n-1$ for $n>s+1$, we have

$$
a_{n} \Delta z_{n}-a_{s} \Delta z_{s}-\frac{M}{p} \sum_{t=s}^{n-1} q_{t} z_{t-l+k} \leq 0 .
$$

Again summing the last inequality from $n-l+k$ to $n-1$ for $s$, we have

$$
z_{n-l+k}-z_{n} \leq \frac{M}{p} z_{n-l+k} \sum_{s=n-l+k}^{n-1} \frac{1}{a_{s}} \sum_{t=s}^{n-1} q_{t}
$$

or

$$
\frac{p}{M} \geq \sum_{s=n-l+k}^{n-1} \frac{1}{a_{s}} \sum_{t=s}^{n-1} q_{t}
$$

which contradicts (2.4). This completes the proof of the theorem.

Theorem 2.2. Assume that $0<\alpha<1$ and $l>k$. If

$$
\begin{gathered}
\limsup _{n \rightarrow \infty} \sum_{s=n-l+k}^{n-1} \frac{1}{a_{s}} \sum_{t=s}^{n-1} q_{t}>0, \\
\sum_{n=n_{0}}^{\infty} q_{n}\left(A_{n-l}+p_{n-l} a_{n-l-k}\right)^{\alpha}=\infty,
\end{gathered}
$$

and for any constant $M_{1}>0$

$$
\sum_{n=n_{0}}^{\infty}\left[M_{1} B_{n+1} q_{n}-\frac{1}{4 a_{n} B_{n+1}}\right]=\infty,
$$

then every solution of equation (1.1) is oscillatory.

Proof. Assume that there exists a nonoscillatory solution $\left\{x_{n}\right\}$ of equation (1.1), say, $x_{n}>0$ and $x_{n-\theta}>0$ for $n \geq N \in \mathbb{N}\left(n_{0}\right)$, where $N$ is chosen so that all three cases of Lemma 2.1 are hold for all $n \geq N$.

Case I: Proceeding as in Case (I) of Theorem 2.1, we obtain $\left\{w_{n}\right\}$ is an eventually positive solution of the inequality

$$
\Delta w_{n}+M q_{n}\left(A_{n-l}+p_{n-l} A_{n-l-k}\right)^{\alpha} w_{n-l}^{\alpha} \leq 0 .
$$


But by Theorem 1 of [10], and (2.19), the inequality (2.21) has no eventually positive solution, a contradiction.

Case II: Define

$$
w_{n}=\frac{a_{n} \Delta z_{n}}{z_{n}}, n \geq N .
$$

Proceeding as in Case (II) of Theorem 2.1, we obtain (2.12) and

$$
\begin{aligned}
\Delta w_{n} & \leq-M q_{n} \frac{z_{n-l}^{\alpha}}{z_{n+1}}-\frac{w_{n}^{2}}{a_{n}} \\
& \leq-M q_{n} z_{n-l}^{\alpha-1}-\frac{w_{n}^{2}}{a_{n}} \\
& \leq-M_{1} q_{n}-\frac{w_{n}^{2}}{a_{n}}, n \geq N,
\end{aligned}
$$

where we have used $\left\{z_{n}\right\}$ is a positive decreasing, $\alpha<1$, and $M_{1}=M z_{N-l}^{\alpha-1}$. The remaining part of the proof is similar to that of Case (II) of Theorem 2.1 and hence the details are omitted.

Case III: Proceeding as in Case (III) of Theorem 2.1, we have

$$
a_{n} \Delta z_{n}-a_{s} \Delta z_{s}-\frac{M}{p^{\alpha}} \sum_{t=s}^{n-1} q_{t} z_{n-l+k}^{\alpha} \leq 0 .
$$

Let $\lim _{n \rightarrow \infty} z_{n}=c=0$. Summing (2.22) from $n-l+k$ to $n-1$ for $s$, we have

$$
z_{n-l+k}-z_{n} \leq \frac{M}{p^{\alpha}} z_{n-l+k} \sum_{s=n-l+k}^{n-1} \frac{1}{a_{s}} \sum_{t=s}^{n-1} q_{t}
$$

or

$$
\frac{z_{n-l+k}}{z_{n-l+k}^{\alpha}} \geq \frac{M}{p^{\alpha}} \sum_{s=n-l+k}^{n-1} \frac{1}{a_{s}} \sum_{t=s}^{n-1} q_{t} .
$$

Since

we have

$$
\frac{z_{n-l+k}}{z_{n-l+k}^{\alpha}}=\left|z_{n-l+k}\right|^{1-\alpha} \text { and } 1-\alpha>0,
$$

$$
\limsup _{n \rightarrow \infty} \sum_{s=n-l+k}^{n-1} \frac{1}{a_{s}} \sum_{t=s}^{n-1} q_{t} \leq 0,
$$

which contradicts (2.18). Next assume that $\lim _{n \rightarrow \infty} z_{n}=c<0$. From (2.18), we claim that

$$
\limsup _{n \rightarrow \infty} \sum_{s=N}^{n-1} \frac{1}{a_{s}} \sum_{t=s}^{n-1} q_{t}=\infty .
$$

In fact from (2.18), there exists a subsequence $\left\{n_{i}\right\}$ and $n_{i+1}-n_{i} \geq l-k$ such that

$$
\sum_{s=n_{i}-l+k}^{n_{i}-1} \frac{1}{a_{s}} \sum_{t=s}^{n_{i}-1} q_{t} \geq b>0,
$$


where $b$ is some constant.

Hence

$$
\begin{aligned}
\lim _{n \rightarrow \infty} \sum_{s=N}^{n-1} \frac{1}{a_{s}} \sum_{t=s}^{n-1} q_{t} & \geq \lim _{j \rightarrow \infty} \sum_{i=1}^{j} \sum_{s=n_{i}-l+k}^{n_{i}-1} \frac{1}{a_{s}} \sum_{t=s}^{n-1} q_{t} \\
& \geq \lim _{j \rightarrow \infty} \sum_{i=1}^{j} \sum_{s=n_{i}-l+k}^{n_{i}-1} \frac{1}{a_{s}} \sum_{t=s}^{n_{i}-1} q_{t} \\
& =\infty,
\end{aligned}
$$

where $n_{j}=\max \left\{n_{i}: n_{i} \leq n\right\}$. From (2.22), we have

$$
\Delta z_{s}+\frac{M z_{n}^{\alpha}}{p^{\alpha} a_{s}} \sum_{t=s}^{n-1} q_{t} \geq 0
$$

Summing the last inequality from $N$ to $n-1$, we obtain

$$
z_{N}-z_{n} \leq \frac{M}{p^{\alpha}} z_{n}^{\alpha} \sum_{s=N}^{n-1} \frac{1}{a_{s}} \sum_{t=s}^{n-1} q_{t}
$$

or

$$
\frac{p^{\alpha} z_{N}}{M z_{n}^{\alpha}} \geq \sum_{s=N}^{n-1} \frac{1}{a_{s}} \sum_{t=s}^{n-1} q_{t}
$$

In view of $c<0, \frac{p^{\alpha} z_{N}}{M z_{n}^{\alpha}}$ has an upper bound, so

$$
\lim _{n \rightarrow \infty} \sum_{s=N}^{n-1} \frac{1}{a_{s}} \sum_{t=s}^{n-1} q_{t}<\infty
$$

which contradicts (2.24). This completes the proof of the theorem.

Theorem 2.3. Let $\alpha>1$. If

$$
\sum_{n=n_{0}}^{\infty} q_{n}\left(1+p_{n-l} \frac{A_{n-l-k}}{A_{n-l}}\right)^{\alpha}=\infty
$$

and

$$
\sum_{n=n_{0}}^{\infty} \frac{1}{a_{n}} \sum_{s=n_{0}}^{n-1} q_{s} B_{s-l}^{\alpha}=\infty,
$$

then every solution $\left\{x_{n}\right\}$ of equation (1.1) is either oscillatory or $\lim _{n \rightarrow \infty} x_{n}=0$.

Proof. Proceeding as in the proof of Theorem 2.2, we see that Lemma 2.1 holds for all $n \geq N \in$ $\mathbb{N}\left(n_{0}\right)$.

Case I: Proceeding as in the proof of Theorem 2.1(Case(I)), we have

$$
\Delta\left(a_{n} \Delta z_{n}\right)+M q_{n}\left(1+p_{n-l} \frac{A_{n-l-k}}{A_{n-l}}\right)^{\alpha} z_{n-l}^{\alpha} \leq 0, n \geq N .
$$


Define $w_{n}=\frac{a_{n} \Delta z_{n}}{z_{n-l}^{\alpha}}$, then $w_{n}>0$, and

$$
\begin{aligned}
\Delta w_{n} & \leq-M q_{n}\left(1+p_{n-l} \frac{A_{n-l-k}}{A_{n-l}}\right)^{\alpha}-\frac{\alpha a_{n+1} \Delta z_{n+1} \Delta z_{n-l}}{z_{n-l}^{\alpha}} \\
& \leq-M q_{n}\left(1+p_{n-l} \frac{A_{n-l-k}}{A_{n-l}}\right)^{\alpha}, n \geq N .
\end{aligned}
$$

Summing the last inequality from $N$ to $n-1$, we obtain

$$
\sum_{s=N}^{n-1} M q_{s}\left(1+p_{s-l} \frac{A_{s-l-k}}{A_{s-l}}\right)^{\alpha}<w_{N}<\infty .
$$

Letting $n \rightarrow \infty$, in the last inequality, we obtain a contradiction to (2.25).

Case II: From Lemma 2.3, we have

$$
z_{n-l}>-B_{n-l} a_{n} \Delta z_{n} \geq-B_{n-l} a_{N} \Delta z_{N} \geq d B_{n-l}
$$

where $d=-a_{N} \Delta z_{N}$.

From equation (1.1), $\left(H_{5}\right)$ and (2.27), we obtain

$$
\Delta\left(-a_{n} \Delta z_{n}\right) \geq M q_{n} d^{\alpha} B_{n-l}^{\alpha}, n \geq N .
$$

Summing the last inequality from $N$ to $n-1$, we have

$$
-a_{n} \Delta z_{n} \geq-a_{N} \Delta z_{N}+M d^{\alpha} \sum_{s=N}^{n-1} q_{s} B_{n-l}^{\alpha} .
$$

Dividing the last inequality by $a_{n}$ and then summing it from $N$ to $n-1$, we obtain

$$
z_{N} \geq z_{N}-z_{n} \geq M d^{\alpha} \sum_{s=N}^{n-1} \frac{1}{a_{s}} \sum_{t=N}^{s-1} q_{t} B_{t-l}^{\alpha} .
$$

Letting $n \rightarrow \infty$ in the last inequality, we obtain

$$
\sum_{n=N}^{\infty} \frac{1}{a_{s}} \sum_{s=N}^{n-1} q_{s} B_{s-l}^{\alpha} \leq z_{N}
$$

a contradiction to $(2.26)$.

Case III. In this case $z_{n}<0$ and $\Delta z_{n}>0$ for all $n \geq N$. Then by Lemma 1 of [12], we see that $\lim _{n \rightarrow \infty} x_{n}=0$. This completes the proof.

\section{Examples}

In this section, we present some examples to illustrate the main results. 
Example 3.1. Consider the second order neutral difference equation

$$
\Delta\left(2^{n} \Delta\left(x_{n}-\frac{1}{2} x_{n-2}\right)\right)+3\left(2^{n}\right) x_{n-3}\left(1+x_{n-3}^{2}\right)=0, n \geq 1 .
$$

Here $a_{n}=2^{n}, p_{n}=\frac{1}{2}, q_{n}=3\left(2^{n}\right), l=3, k=2, \alpha=1$, and $M=1$. Since $A_{n}=1-\frac{1}{2^{n-1}}$ and $B_{n}=\frac{1}{2^{n-1}}$, it is easy to see that all conditions of Theorem 2.1 are satisfied and hence every solution of equation (3.1) is oscillatory. In fact $\left\{x_{n}\right\}=\left\{(-1)^{n}\right\}$ is one such oscillatory solution of equation (3.1).

Example 3.2. Consider the second order neutral difference equation

$$
\Delta\left(2^{n} \Delta\left(x_{n}-\frac{1}{2^{n}} x_{n-1}\right)\right)+2^{1-\frac{n}{3}}\left(15\left(4^{n}\right)+3\left(2^{n}\right)\right) x_{n-3}^{\frac{1}{3}}=0, n \geq 1 .
$$

Here $a_{n}=2^{n}, p_{n}=\frac{1}{2^{n}}, q_{n}=2^{1-\frac{n}{3}}, l=3, k=1, \alpha=\frac{1}{3}$, and $M=1$. Since $A_{n}=1-\frac{1}{2^{n-1}}$ and $B_{n}=$ $\frac{1}{2^{n-1}}$, it is easy to see that all conditions of Theorem 2.2 are satisfied and hence every solution of equation (3.2) is oscillatory. In fact $\left\{x_{n}\right\}=\left\{(-1)^{3 n} 2^{n}\right\}$ is one such oscillatory solution of equation (3.2).

Example 3.3. Consider the second order neutral difference equation

$$
\Delta\left(n(n+1) \Delta\left(x_{n}-\frac{1}{2} x_{n-1}\right)\right)+\frac{(n-2)^{3}}{n(n-1)} x_{n-2}^{3}=0, n \geq 3 .
$$

Here $a_{n}=n(n+1), p_{n}=\frac{1}{2}, q_{n}=\frac{(n-2)^{3}}{n(n-1)}, l=2, k=1, \alpha=3$, and $M=1$. Since $A_{n}=\frac{n-3}{3 n}$ and $B_{n}=\frac{1}{n}$, it is easy to see that all conditions of Theorem 2.3 are satisfied and so any solution of equation (3.3) is either oscillatory or tends to zero as $n \rightarrow \infty$.. In fact $\left\{x_{n}\right\}=\left\{\frac{1}{n}\right\}$ is one such solution of equation (3.3) of the latter type.

We conclude this paper with the following remark.

Remark 3.1. It would be interesting to improve the result of Theorem 2.3 to similar that of Theorem 2.1.

\section{Acknowledgement}

The author E.Thandapani gratefully acknowledges the University Grants Commission(India) for awarding Emeritus Fellowship(No.F.6-6/2013-14/EMERITUS-2013-14-GEN-2747/(SA-II)) to carry out this research.Further the authors thank the reviewer for his/her suggestions which improved the content of the paper. 


\section{References}

[1] R. P. Agarwal, Difference Equations and Inequalities, Second Edition, Marcel Dekker, New York, 2000.

[2] R. P. Agarwal, M. Bohner S. R. Grace, and D. O'Regan, Discrete Oscillation Theory, Hindawi Publ. Corp., New York, 2005.

[3] R. P. Agarwal, S. R. Grace and E. Thandapani, Oscillatory and nonoscillatory behavior of second order neutral delay difference equations, Math. Comp. Model., 24 (1996), 5-11.

[4] W.T. Li, Oscillation of higher order neutral nonlinear difference equations, Appl. Math. Lett., 11 (1998), 1-8.

[5] I. Gyori and G. Ladas, Oscillation Theory of Delay Differential equations With Applications, Clarendon press, Oxford, 1991.

[6] S. H. Saker, Oscillation Theory of Delay Differential and Difference Equations: Second and Third Orders, Verlag dr Muler, Germany, 2010.

[7] S. H. Saker and D. O'Regan, New oscillation criteria for second order neutral functional dynamic equations via the generalized Riccati substitution, Commu. Nonlin. Sci. Numer. Simu., 16 (2011), 423-434.

[8] S. H. Saker and D. O'Regan, New oscillation criteria for second order nonlinear neutral functional dynamic equations on time scales via Riccati substitution, Hirishima J. Math., 42 (2012), 77-98.

[9] Y. G. Sun and S. H. Saker, Oscillation for second order nonlinear neutral delay difference equations, Appl. Math. Comput., 163 (2005), 909-918.

[10] X. H. Tang and Y. Liu, Oscillation for nonlinear delay difference equations, Tamkang J. Math., 32 (2001), 275280.

[11] E. Thandapani, P. Sundaram, J. R. Graef and P. W. Spikes, Asymptotic properties of solutions of nonlinear second order neutral delay difference equations, Dynamic Sys. Appl., 4(1995), 125-136.

[12] E. Thandapani, V. Balasubramanian and J. R. Graef, Oscillation criteria for second order neutral difference equations with negative neutral term, Inter. J. Pure. Appl. Math., 87 (2013), 283-292.

[13] E. Thandapani, Z. Liu, R. Arul and P. S. Raja, Oscillation and asymptotic behavior of second order difference equations with nonlinear neutral terms, Appl. Math. E-Notes, 4 (2004), 59-67.

[14] E. Thandapani and K. Mahalingam, Necessary and sufficient conditions for oscillation of second order neutral delay difference equations, Tamkang J. Math., 34 (2003), 137-145.

[15] D. M. Wang and Z. T. Xu, Oscillation of second order quasilinear neutral delay difference equations, Acta. Math. Appl. Sinica., 27 (2011), 93-104.

[16] A. Zafer and R. S. Dahiya, Oscillation of a neutral difference equations, Appl. Math. Lett., 6(1993), 71-74.

[17] Z. Zhou, J. Yu and G. Lei, Oscillation for even order neutral difference equations, Korean J. Comput. Appl. Math., 7 (2000), 601-610.

Ramanujan Institute for Advanced Study in Mathematics, University of Madras, Chennai -600 005, India.

E-mail: ethandapani@yahoo.co.in

Ramanujan Institute for Advanced Study in Mathematics, University of Madras, Chennai -600 005, India.

E-mail: d.seghar@gmail.com

Academia Militar, Departamento de Ciencias Exactas e Naturais, Av. Conde Castro Guimaraes, 2720-113 Amadora, Portugal.

E-mail: sandra.pinelas@gmail.com 